\title{
THE ASYMPTOTIC BEHAVIOR OF TOEPLITZ DETERMINANTS GENERATED BY THE LAURENT COEFFICIENTS OF A MEROMORPHIC FUNCTION*
}

\author{
ADHEMAR BULTHEEL $\dagger$
}

\begin{abstract}
By combining known results on the asymptotic behavior of Hankel and Toeplitz determinants we obtain expressions for det $\left(f_{m+i-j}\right)_{i, j=0}^{n}$ as $n \rightarrow \infty$ and $m=0, \pm 1, \pm 2, \cdots$. The $f_{k}$ are Laurent coefficients of a meromorphic function. These expressions contain the zeros of the meromorphic function and are therefore interesting in proving results on the convergence properties of Laurent-Padé approximants.
\end{abstract}

Key word. Toeplitz determinants

AMS(MOS) subject classifications. 47B35, 41 A60

1. Introduction. The $q d$-algorithm of Rutishauser [11] or the $\pi \zeta$-algorithm [5] are known to construct a table of numbers, given the Taylor series of a meromorphic function. By considering the column limits of these tables, we can compute the poles of that function. The main tool in the proof of these results is the study of the asymptotic behavior of Hankel determinants constructed with the Taylor series coefficients. By using a symmetry property of the tables constructed by the above algorithms for a Taylor series and its inverse one finds that row limits of these tables allow zeros of that function to be computed. If the meromorphic function is given by a Laurent series in a certain annular region, centered around the origin, it is possible to construct by the same algorithms a bi-infinite table of numbers [6, p. 62]. As in the case of a Taylor series one can prove that the downward limits can be used to compute the poles of the function around infinity. The upward limits of the columns can be used to find the poles around the origin. In [8] this was shown in the context of two point Padé approximants for an algorithm developed by McCabe and Murphy [10]. This algorithm is of the same type and closely related to the $q d$ - and $\pi \zeta$-algorithms. We call it the FG-algorithm, referring to the notation used in [9]. The method of proof is based on an additive splitting of the Laurent series,

$$
f(z)=\sum_{-\infty}^{\infty} f_{k} z^{k}=\sum_{0}^{\infty} f_{k} z^{k}+\sum_{1}^{\infty} f_{-k} z^{-k}
$$

The downward column limits depend only on the coefficients in the first sum. The upward column limits depend only on the coefficients in the second sum. Consequently the theory developed for a Taylor series can again be used. It is to be expected that the row limits will give information about the zeros. This is indeed the case [2], [3]. These row limits depend, however, on the complete Laurent series and there is no obvious symmetry property that can derive the results from the previous theory. It turns out that instead of the asymptotic behavior of the Hankel determinant $\operatorname{det}\left[f_{m+i+j}\right]_{i, j=0}^{n}$ as $m \rightarrow \infty$ we now have to investigate its behavior as $n \rightarrow \infty$, or what is the same, the behavior of Toeplitz determinants $\operatorname{det}\left[f_{m+i-j}\right]_{i, j=0}^{n}$ as $n \rightarrow \infty$. The latter is the subject of this paper. It will be clear at the end that the theory for a Taylor series can still be used but now for a multiplicative splitting of $f: f(z)=f_{+}(z) f_{-}(z)$, where

* Received by the editors January 11, 1984, and in final revised form June 5, 1984.

$\dagger$ Department of Computer Science, Katholieke Universiteit Leuven, Celestijnenlaan 200A, B-3030 Leuven, Belgium. 
$f_{+}$is analytic around the origin and $f_{-}(z)$ is analytic around infinity. Several expressions for the asymptotics of Toeplitz determinants exist in the literature. Usually they are constructed from the Fourier coefficients of a function that has certain properties. We shall use these and adapt them to our situation. In view of the application it is important that the zeros of the function are introduced in the expressions. It should be noted that for rational functions and under certain restricting conditions an explicit expression for Toeplitz determinants was given in [4].

We do not include the proofs on the row limits of the tables constructed by the rhombus rules because these were given elsewhere and because we think the expressions may be interesting in their own right.

2. Notation and main results. Let $f(z)$ be meromorphic in $\mathbb{C}_{0}=\mathbb{C} \backslash\{0\}$ and suppose there exist $r$ and $R$ such that

$$
f(z)=\sum_{-\infty}^{\infty} f_{k} z^{k}
$$

is a Laurent series of $f(z)$, convergent in the annulus $r<|z|<R$.

We suppose further that

$$
f(z)=G_{\rho}(\tilde{f}) f_{+}(z) f_{-}(z) z^{\kappa}
$$

with

$$
\tilde{f}(z)=z^{-\kappa} f(z)
$$

and

$$
G_{\rho}(\tilde{f})=\exp \left[\frac{1}{2 \pi} \int_{0}^{2 \pi} \log \tilde{f}\left(\rho e^{i \theta}\right) d \theta\right], \quad r<\rho<R
$$

and

$$
\begin{aligned}
& f_{+}(z)=\prod_{k \in Z^{+}}\left(1-\frac{z}{\zeta_{k}^{+}}\right) / \prod_{k \in P^{+}}\left(1-\frac{z}{\pi_{k}^{+}}\right), \\
& f_{-}(z)=\prod_{k \in Z^{-}}\left(1-\frac{\zeta_{k}^{-}}{z}\right) / \prod_{\pi \in P^{-}}\left(1-\frac{\pi_{k}^{-}}{z}\right),
\end{aligned}
$$

where the zeros and poles are numbered such that

$$
\begin{aligned}
& \cdots \leqq\left|\zeta_{2}^{-}\right| \leqq\left|\zeta_{1}^{-}\right| \leqq r<R \leqq\left|\zeta_{1}^{+}\right| \leqq\left|\zeta_{2}^{+}\right| \leqq \cdots \\
& \cdots \leqq\left|\pi_{2}^{-}\right| \leqq\left|\pi_{1}^{-}\right| \leqq r<R \leqq\left|\pi_{1}^{+}\right| \leqq\left|\pi_{2}^{+}\right| \leqq \cdots
\end{aligned}
$$

We suppose that $\zeta_{k}^{+}, k \in Z^{+}$, are all finite and we set $\zeta_{k}^{+}=\infty$ if $k>\# Z^{+}$and similarly for $\pi_{k}^{+}$. Also $\zeta_{k}^{-} \neq 0$ for $k \in Z^{-}$and $\zeta_{k}^{-}=0$ for $k>\# Z^{-}$and similarly for $\pi_{k}^{-}$.

$f_{+}$and $f_{-}$are supposed to be irreducible. $\kappa$ of $(2.2)$ is the winding number

$$
\kappa=\kappa_{\rho}=\operatorname{ind}_{\rho}(f)=\frac{1}{2 \pi}\left[\arg f\left(\rho e^{i \theta}\right)\right]_{0}^{2 \pi} .
$$

Note that $\operatorname{ind}_{\rho}\left(f_{+}\right)=\operatorname{ind}_{\rho}\left(f_{-}\right)=\operatorname{ind}_{\rho}(\tilde{f})=0$. For a rational function $f$, ind $(f)=$ the number of zeros in $|z|<\rho$-the number of poles in $|z|<\rho$. With the series (2.1) we associate the Toeplitz determinants

$$
T_{n}^{(m)}(f)=\operatorname{det}\left(f_{m+i-j}\right)_{i, j=0}^{n}, \quad m, n=0, \pm 1, \pm 2, \cdots .
$$


Then for $n \rightarrow \infty$, we have the following asymptotic expansions:

THEOREM 1. With the notation just introduced we have for $n \rightarrow \infty$ :

$$
\begin{aligned}
T_{n}^{(m)}(f)= & c\left[G_{\rho}(\tilde{f})\right]^{n+1}\{1+o(1)\} \quad \text { if } \quad m=\kappa, \\
T_{n}^{(m)}(f)= & c\left[G_{\rho}(\tilde{f})\right]^{n+1}\left[\left(-\zeta_{1}^{+}\right)\left(-\zeta_{2}^{+}\right) \cdots\left(-\zeta_{m-\kappa}^{+}\right)\right]^{-n} \\
& \cdot\left\{1+O\left(\left|\zeta_{m-\kappa}^{+} / \sigma\right|^{n}\right)\right\}
\end{aligned}
$$

if $0<m-\kappa<\# Z^{+}+1$ and if $\sigma$ satisfying $\left|\zeta_{m-\kappa}^{+}\right|<\sigma<\left|\zeta_{m-\kappa+1}^{+}\right|$exists.

$$
\begin{aligned}
T_{n}^{(m)}(f)= & c\left[G_{\rho}(\tilde{f})\right]^{n+1}\left[\left(-\zeta_{1}^{-}\right)\left(-\zeta_{2}^{-}\right) \cdots\left(-\zeta_{\kappa-m}^{-}\right)\right]^{n} \\
& \cdot\left\{1+O\left(\left|\sigma_{\kappa-m}^{-}\right|^{n}\right)\right\}
\end{aligned}
$$

if $0<\kappa-m<\# Z^{-}+1$ and if $\sigma$ satisfying $\left|\zeta_{\kappa-m+1}^{-}\right|<\sigma<\left|\zeta_{\kappa-m}^{-}\right|$exists.

Here and in the following $c$ is a constant not depending on $n$. It is seen from this that the asymptotic behavior of $T_{n}^{(m)}(f)$ is up to a factor completely defined by $f_{+}$or $f_{-}$. We have thus the following theorem.

THEOREM 2.

$$
T_{n}^{(m)}(f) \sim T_{n}^{(m-\kappa)}\left(f_{+}\right)\left[G_{\rho}(\tilde{f})\right]^{n+1}
$$

where $m \geqq \kappa, \tilde{f}(z)=z^{-\kappa} f(z)$, and

$$
T_{n}^{(m)}(f) \sim T_{n}^{(\kappa-m)}\left(\hat{f}_{-}\right)\left[G_{\rho}(\tilde{f})\right]^{n+1}
$$

where $m \leqq \kappa, \hat{f}_{-}(z)=f_{-}(1 / z), \tilde{f}(z)=z^{-\kappa} f(z)$.

As a special case we reformulate Theorem 1 for a rational function $f(z)$.

THEOREM 3. Suppose $f(z)$ is a rational function with irreducible form

$$
f(z)=K \cdot z^{l} \cdot \frac{\left(z-\zeta_{1}\right)\left(z-\zeta_{2}\right) \cdots\left(z-\zeta_{N}\right)}{\left(z-\pi_{1}\right)\left(z-\pi_{2}\right) \cdots\left(z-\pi_{M}\right)}
$$

Let $f(z)=\sum_{-\infty}^{\infty} f_{k} z^{k}$ in $r<|z|<R$ and $f(z) \neq 0$ on $|z|=\rho$ with $r<\rho<R$. We order poles and zeros as follows

$$
\begin{aligned}
& 0<\left|\zeta_{N}\right| \leqq\left|\zeta_{N-1}\right| \leqq \cdots \leqq\left|\zeta_{1}\right|<\infty \\
& 0<\left|\pi_{M}\right| \leqq\left|\pi_{M-1}\right| \leqq \cdots \leqq\left|\pi_{P+1}\right|<\rho<\left|\pi_{P}\right| \leqq\left|\pi_{P-1}\right| \leqq \cdots \leqq\left|\pi_{1}\right|<\infty
\end{aligned}
$$

$M-P$ is the number of nonzero poles in $|z|<\rho$. Then for $0 \leqq k-m \leqq N$, where $k=N-M+P+l$, we have

$$
\begin{aligned}
T_{n}^{(m)}(f)= & c K^{n}\left[\left(-\zeta_{1}\right)\left(-\zeta_{2}\right) \cdots\left(-\zeta_{k-m}\right)\right]^{n}\left[\left(-\pi_{1}\right)\left(-\pi_{2}\right) \cdots\left(-\pi_{P}\right)\right]^{-n} \\
& \cdot\left\{1+O\left(\left|\frac{\zeta_{k-m+1}}{\sigma}\right|^{n}\right)\right\}, \quad n \rightarrow \infty,
\end{aligned}
$$

where it is understood that $\zeta_{0}$ and $\zeta_{N+1}$ are arbitrary and defined by

$$
0<\zeta_{N+1}<\left|\zeta_{N}\right| \text { and }\left|\zeta_{1}\right|<\zeta_{0}<\infty
$$

and on condition $\sigma$ exists satisfying $\left|\zeta_{k-m+1}\right|<\sigma<\left|\zeta_{k-m}\right|$.

We prove Theorem 1 by taking results on the asymptotic behavior of Toeplitz determinants from the literature. By combining them and using some elementary transformations we get the desired results. 
3. Proof of Theorem 1. We shall start by eliminating the geometric mean $G_{\rho}(\tilde{f})$ from the formula. To do this use the simple observation given in

LEMMA 1.

$$
T_{n}^{(m)}(c \cdot f)=c^{n+1} T_{n}^{(m)}(f) .
$$

This allows us to set for the moment $G_{\rho}(\tilde{f})=1$ without loss of generality. The next step is to solve the problem for $\kappa=0$ and for $\rho=1$, so that the $f_{k}$ may be considered to be the Fourier coefficients of $f(z)$.

We first quote the following theorem which was proved in a more general context in [1]. For our case it reads as follows.

TheOREM 4. Let $\rho=1, G_{\rho}(f)=1$ and ind $_{\rho}(f)=0$. Then, as $n \rightarrow \infty$, we have

$$
\begin{aligned}
& T_{n}^{(0)}(f)=c\{1+o(1)\}, \\
& T_{n}^{(m)}(f)=c(-1)^{(n+m) m}\left\{T_{m-1}^{(n+1)}\left(f_{+}^{-1}\right)+o(1)\right\}, \quad m>0, \\
& T_{n}^{(-m)}(f)=c(-1)^{(n+m) m}\left\{T_{m-1}^{(-n-1)}\left(f_{+} / f_{-}\right)+o(1)\right\}, \quad m>0 .
\end{aligned}
$$

In this case $c$ is even specified to be

$$
\exp \left(\sum_{k=1}^{\infty}\left(k(\log f)_{-k}(\log f)_{k}\right)\right)
$$

with $\left\{(\log f)_{k}\right\}_{k=-\infty}^{\infty}$ the Fourier coefficients of $\log f$.

We shall now derive expressions for $T_{m-1}^{(n+1)}\left(f_{+}^{-1}\right)$ and $T_{m-1}^{(-n-1)}\left(f_{+} / f_{-}\right)$. This will be done via known asymptotic expressions for finite Toeplitz determinants. The basic result is classical and given in [7, p. 596]. Similar results are obtained in [5, p. 45].

THeOREM 5. Let $f(z)=\sum_{0}^{\infty} f_{k} z^{k}$ be the Taylor series of a meromorphic function, analytic at the origin, with poles $\pi_{k}$, ordered as

$$
0<\left|\pi_{1}\right| \leqq\left|\pi_{2}\right| \leqq \cdots \leqq\left|\pi_{N}\right| \text {. }
$$

Let $\pi_{N+1}=\infty$ and $f_{k}=0$ for $k<0$. Then

$$
T_{m-1}^{(n)}(f)=c\left(\pi_{1} \cdots \pi_{m}\right)^{-n}\left[1+O\left(\left|\pi_{m} / \sigma\right|^{n}\right)\right], \quad n \rightarrow \infty,
$$

if $0<m<N+1$ and $\sigma$ exists satisfying $\left|\pi_{m}\right|<\sigma<\left|\pi_{m+1}\right|$.

To extend this result to Toeplitz determinants of a Laurent series we use the following corollary.

Corollary 1. Let $f(z)$ and its Laurent series be as in (2.1)-(2.6). Then

$$
T_{m-1}^{(n)}(f)=c\left(\pi_{1}^{+} \cdots \pi_{m}^{+}\right)^{-n}\left[1+O\left(\left|\pi_{m}^{+} / \sigma\right|^{n}\right)\right], \quad n \rightarrow \infty,
$$

if $0<m<\# P^{+}+1$ and $\left|\pi_{m}^{+}\right|<\sigma<\left|\pi_{m+1}^{+}\right|$, and

$$
T_{m-1}^{(-n)}(f)=c\left(\pi_{1}^{-} \pi_{2}^{-} \cdots \pi_{m}^{-}\right)^{n}\left[1+O\left(\left|\sigma / \pi_{m}^{-}\right|^{n}\right)\right], \quad n \rightarrow \infty,
$$

if $0<m<\# P^{-}+1$ and $\left|\pi_{m+1}^{-}\right|<\sigma<\left|\pi_{m}^{-}\right|$.

Proof. Split $f(z)$ as $f(z)=g(z)+\hat{g}(z)$ with

$$
g(z)=\sum_{0}^{\infty} f_{k} z^{k} \quad \text { and } \quad \hat{g}(z)=\sum_{1}^{\infty} f_{-k} z^{-k} .
$$

Clearly $g(z)$ defines a function with poles $\pi_{k}^{+}, k \in P^{+}$, arranged as

$$
\rho<\left|\pi_{1}^{+}\right| \leqq\left|\pi_{2}^{+}\right| \leqq \cdots .
$$

Because $T_{m-1}^{(n)}(f)$ for $n \geqq 0$ depends only on the coefficients $f_{0}, f_{1}, \cdots$ we have $T_{m-1}^{(n)}(f)=$ $T_{m-1}^{(n)}(g)$. Thus Theorem 5 applies, and this proves the first part of the corollary. 
Set $h(z)=\hat{g}(1 / z)$ : This function has poles $\left(\pi_{k}^{-}\right)^{-1}, k \in P^{-}$, ordered like

$$
\rho^{-1}<\left|\pi_{1}^{-}\right|^{-1} \leqq\left|\pi_{2}^{-}\right|^{-1} \leqq \cdots .
$$

$T_{m-1}^{(-n)}(f)$ depends only on $f_{-1}, f_{-2}, \cdots$ if $n \geqq m$. Since $T_{m-1}^{(-n)}(f)=T_{1-m}^{(n)}(h)=T_{m-1}^{(n)}(h)$, we can again apply Theorem 5 which gives the second part of the statement.

With this corollary we can prove that Theorem 1 is true for $\rho=1$ and $\kappa=\operatorname{ind}_{\rho}(f)=$ 0 . The general case can be shown by using the trivial transformations given in the following lemma.

LEMMA 2. With the notation of $\S 2$, set

$$
\tilde{f}(z)=z^{-\kappa} f(z) \quad \text { and } \quad g(z)=\tilde{f}(\rho z)=\sum_{-\infty}^{\infty} g_{k} z^{k}
$$

for $r^{\prime}<|z|<R^{\prime}$ with $g_{k}=\rho^{k} f_{k+\kappa}, r^{\prime}=r / \rho<1$ and $R^{\prime}=R / \rho>1$. Then we have:

(1) $G_{\rho}(\tilde{f})=G_{1}(g)$,

(2) $T_{n}^{(m)}(f)=T_{n}^{(m-\kappa)}(\tilde{f})=\rho^{-(m-\kappa) n} T_{n}^{(m-\kappa)}(g)$,

(3) If $\tau$ is a pole (zero) of $f$, then $\tau / \rho$ is a pole (zero) of $g(z)$, with a possible exception for $\tau=0$ or $\tau=\infty$.

4. Proof of Theorem 3. Note that $f(z)$ can be written in the form (2.1) if we take

$$
\begin{array}{lllllll}
\zeta_{1}, & \zeta_{2}, & \cdots, & \zeta_{\#} Z^{+}, & \zeta_{\# Z^{+}+1}, & \cdots, & \zeta_{N}, \\
\| & \| & & \| & \| & & \| \\
\zeta_{\#}^{+} Z^{+}, & \zeta_{\# Z^{+}-1}^{+}, & \cdots, & \zeta_{1}^{+}, & \zeta_{1}^{-}, & \cdots, & \zeta_{\# Z^{-}}
\end{array}
$$

with $\left|\zeta_{i}^{+}\right|>\rho$ for $i=1,2, \cdots, \# Z^{+}$and $\left|\zeta_{i}^{-}\right|<\rho$ for $i=1,2, \cdots, \# Z^{-}$.

Similarly for the poles.

$$
\begin{array}{lllllll}
\pi_{1}, & \pi_{2}, & \cdots, & \pi_{P}, & \pi_{P+1}, & \cdots, & \pi_{M}, \\
\| & \| & & \| & \| & & \| \\
\pi_{\# P^{+}}^{+}, & \pi_{\# P^{+}-1}^{+}, & \cdots, & \pi_{1}^{+}, & \pi_{1}^{-}, & \cdots, & \pi_{\# P^{-}}^{-}
\end{array}
$$

$\left(\# P^{+}=P\right.$ and $\# P^{-}=M-P$ ).

Clearly $\kappa=\# Z^{-}-\# P^{-}+l$, and

$$
G_{\rho}(\tilde{f})=K \cdot \frac{\left(-\zeta_{1}^{+}\right) \cdots\left(-\zeta_{\# Z^{+}}^{+}\right)}{\left(-\pi_{1}^{+}\right) \cdots\left(-\pi_{\# P^{+}}^{+}\right)} .
$$

Theorem 3 now directly follows from Theorem 1 if you observe that $0<m-\kappa<$ $\# Z^{+}+1$ is equivalent with $0 \leqq k-m<\# Z^{+}, 0<\kappa-m<\# Z^{-}+1$ is equivalent with $\# Z^{+}<k-m \leqq N$ and $k-m=\# Z^{+}$is equivalent with $m=\kappa$.

5. Conclusion. With the multiplicative splitting for $f$ defined in (2.2)-(2.6), it is shown that the asymptotic behavior of the Toeplitz determinants $T_{n}^{(m)}(f)$ are mainly defined by $T_{n}^{(m-\kappa)}\left(f_{+}\right)$or $T_{n}^{(\kappa-m)}\left(\hat{f}_{-}\right)$depending on $m$ being larger or smaller than $\kappa$. With these formulas it is possible to extend the results for a rational function given in [2] to the general meromorphic case.

\section{REFERENCES}

[1] A. BötTCHER AND B. Silbermann, The asymptotic behavior of Toeplitz determinants for generating functions with zeros of integral orders, Math. Nachr., 102 (1981), pp. 70-105.

[2] A. Bultheel, Zeros of a rational function defined by its Laurent series, in Padé Approximation and its Applications, Bad Honnef 1983, H. Werner and H. J. Bünger, eds., Springer-Verlag, Berlin, 1984, pp. $34-48$. 
[3] A. BUltheel, Quotient-difference relations in connection with AR filtering, Proc. ECCTD '83, VDEVerlag, Berlin, 1983, pp. 395-399.

[4] K. M. DAY, Toeplitz matrices generated by an arbitrary rational function, Trans. Amer. Math. Soc., 206 (1975), pp. 224-245.

[5] W. B. GRAGG, The Padé table and its relation to certain algorithms of numerical analysis, SIAM Rev., 14 (1972), pp. 1-62.

[6] - Laurent, Fourier, and Chebyshev-Padé tables, in Padé and Rational Approximation, E. B. Saff and R. S. Varga, eds., Academic Press, New York, 1977, pp. 61-72.

[7] P. Henrici, Applied and Computational Complex Analysis, Vol. 1, John Wiley, New York, 1974.

[8] W. B. Jones ANd A. MAGnus, Computation of poles of two-point Padé approximants and their limits, J. Comp. Appl. Math., 6 (1980), pp. 105-119.

[9] W. B. Jones AND W. J. Thron, Continued Fractions, Analytic Theory and Applications, Academic Press, New York, 1980.

[10] J. N. MCCABE AND J. A. MURPHY, Continued fractions which correspond to power series expansions at two points, J. Inst. Math. Appl., 17 (1976), pp. 233-247.

[11] H. Rutishauser, Der quotienten-differenzen-Algorithmus, Z. Angew Math. Phys., 5 (1954), pp. $233-251$. 